\title{
Myelographic diagnosis and results of surgical treatment of caudal cervical spondylomyelopathy in dogs: a retrospective study (2000-2010)
}

\author{
Robert Srnec ${ }^{1}$, Pavel Proks², Petra Fedorová ${ }^{1}$, Ladislav Stehlík ${ }^{2}$, \\ Milan Dvoř́k ${ }^{1}$, Alois Nečas ${ }^{1}$ \\ ${ }^{1}$ University of Veterinary and Pharmaceutical Sciences Brno, Faculty of Veterinary Medicine, Small Animal \\ Clinic, Department of Surgery and Orthopaedics, ${ }^{2}$ Department of Diagnostic Imaging, Brno, Czech Republic \\ Received May 14, 2012 \\ Accepted September 19, 2012
}

\begin{abstract}
The outcomes of six different surgical techniques of caudal cervical spondylomyelopathy treatment in dogs with static and dynamic spinal cord compressions were assessed. Out of 425 dogs with cervical spine diseases, caudal cervical spondylomyelopathy was diagnosed in 69 dogs. Dynamic myelographic study was performed in 48 of them as a diagnostic method necessary for choosing the appropriate technique of surgical treatment. Twenty-five out of the 48 dogs underwent surgery. The best results of the surgical treatment of static compression were achieved with a ventral slot ( $89 \%$ of the nine surgical interventions) with partial improvement $48 \mathrm{~h}$ after surgery and marked improvement 8 weeks after surgery. For dynamic compressions, vertebral traction and stabilization with intervertebral washer plus vertebral body screws and polymethylmethacrylate bridge was found to be the most successful surgical treatment with significant improvement in $62 \%$ of the thirteen surgical interventions 8 weeks after surgery. In 5 dogs $(20 \%)$, two intervertebral spaces with caudal cervical spondylomyelopathy were surgically treated. Recurrence was observed in 6 dogs $(24 \%)$ after the mean period of 20 months (5 to 44 months) after surgery. Direct ventral slot decompression could be recommended for clinical cases of the caudal cervical spondylomyelopathy with static compression, and intervertebral washer plus vertebral body screws and polymethylmethacrylate bridge for dogs with dynamic compression, respectively. Our findings refer to the results of surgical treatment in a relatively large number of dogs with caudal cervical spondylomyelopathy and can help surgeons to choose an effective surgical method of treatment in dogs with wobbler syndrome based on dynamic myelographic study findings.
\end{abstract}

Wobbler syndrome, canine, neck surgery, spinal cord compression

Caudal cervical spondylomyelopathy (CCSM), the synonym for wobbler syndrome, is a relatively common disease in large and giant breed dogs (Sharp and Wheeler 2005). There are two different groups of dogs with wobbler syndrome. Firstly, it affects juvenile giant breed dogs (typically the Great Dane) with the congenital absolute osseous compression causing a narrowing of the spinal canal or with vertebral malarticulation. This osseousassociated wobbler syndrome commonly affects more intervertebral spaces and sites of the lesions often lead to multiple serious compressions (Da Costa et al. 2012). Secondly, it affects middle-aged large breed dogs (typically the Doberman pinscher) with congenital asymptomatic relative spinal canal stenosis (predisposition factor) causing subsequent degenerative changes of the intervertebral disc and surrounding soft tissues. The second type of CCSM is also called the disc associated wobbler syndrome (Da Costa 2010). The basic step in diagnosis is well performed clinical examination taking into consideration medical history and personal data (breed predisposition, age and sex, duration of problems, degree of neurological signs); however, treatment is the mostly dependent to results of diagnostic imaging examination (Sharp et al. 1992). Despite the available examination on computed tomography (CT) or magnetic resonance imaging (MRI), myelographic examination is still the one most widely used (Robertson and Thrall 2011). Dynamic myelographic study is a fast and accessible method which uses stress projections that help specify the dynamics of

Address for correspondence:

MVDr. Robert Srnec

Department of Surgery and Orthopaedics

Small Animal Clinic, Faculty of Veterinary Medicine

University of Veterinary and Pharmaceutical Sciences Brno

Palackeho tř. 1/3, 61242 Brno, Czech Republic

Phone: +420541562342, +420775385533

Fax: +420 541562344

E-mail: srnecr@vfu.cz

http://actavet.vfu.cz/ 
compression depending on the positioning of the cervical spine. Thus way we differentiate static or dynamic compression. In dynamic lesions it is important to evaluate the changing of compression in traction (traction responsive or non-responsive) for choosing the best technique of surgery (McKee and Sharp 2002). Prognosis of CCSM significantly differs not only depending on the type of lesion and duration of the neurological signs but also on correct evaluation of myelography and the subsequent choice of the method of treatment. It can be either conservative or surgical method. Surgical methods include methods of direct decompression (ventral, dorsal, sporadically lateral) or indirect decompression (traction and stabilisation).

The aim of this retrospective clinical study is to characterize types of lesions based on the dynamic myelographic study and to assess the results of surgical treatment of dogs with CCSM depending on the selected surgical method.

\section{Materials and Methods}

Data from the records of the Department of Surgery and Orthopaedics of the Small Animal Clinic, Faculty of Veterinary Medicine, University of Veterinary and Pharmaceutical Sciences Brno (SAC UVPS Brno) in the period between January 2000 and December 2010 were used to analyze dogs with CCSM. In the group of dogs with neurological findings, the frequency of CCSM was determined. We collected data concerning the breed, sex, age and weight. Basic clinical and neurological examination of dogs was performed according to Garosi (2004). Dogs were divided into 6 groups based on the severity of neurological findings characterized using a modified scale (grade 0-5) of neurological dysfunctions (Table 1) (McKee et al. 1999).

Table 1. Modified scale of neurological dysfunction in dogs (McKee et al. 1999).

Grade 0 Healthy patients with no clinically apparent neurological problems

Grade 1 Neck pain without leg ataxia

Grade 2 Mild ataxia of hind limbs with no neurological deficit in forelimbs

Grade 3 Moderate ataxia of hind limbs with/without neurological deficit in forelimbs

Grade 4 Severe ataxia of hind limbs with/without neurological deficit in forelimbs

Grade 5 Very severe ataxia with inability of movement without help, tetraplegy

Plain radiographs and contrast myelography of the cervical spine in latero-lateral and ventro-dorsal projection were then performed in general anaesthesia. Dogs without complete dynamic myelographic examination were excluded from the reference group. Depending on the myelographic study, we evaluated compression of the spinal canal, the site of compression (intervertebral space), number of lesions (simple, dual, multiple), location of compression within the spinal canal (ventral, dorsal, lateral, combined "hour-glass" compression) and the type of compression (static or dynamic).

Results were evaluated only in dogs surgically treated for CCSM. A total of six methods of surgery of CCSM were used in the reference group: 1 - direct decompression with the ventral slot method (SLOT), 2 - indirect decompression using intervertebral washer (IVW) plus vertebral body screws (BS) and polymethylmethacrylate (PMMA) bridge (IVW+BS-PMMA), 3 - indirect decompression using intervertebral washer and cancellous autograft (CAG) (IVW and CAG), 4 - indirect decompression using intervertebral washer plus buttress plate (BP) and cancellous autograft (IVW+BP and CAG), 5 - indirect decompression using buttress plate and cancellous autograft (BP and CAG), 6 - indirect decompression using smooth pins and PMMA bridge (P-PMMA). We noted the type of performed surgery, evaluation of clinical finding before the surgery, $48 \mathrm{~h}$ after the surgery and 8 weeks after the surgery, and the recovery time from surgery to final clinical status (in weeks). Long-term monitoring of dogs included life expectancy after the surgery and the monitoring of recurrences of CCSM after surgical treatment (in weeks). Twenty-five dogs (52.1\% CCSM completely detected with myelography) were treated surgically; in 5 dogs the surgery was performed in two intervertebral spaces simultaneously. A total of 30 intervertebral spaces with CCSM $(\mathrm{n}=30)$ were assessed. The frequencies of different surgical methods were: SLOT $(n=10)$, IVW+BS-PMMA $(n=13)$, IVW and CAG $(n=4), I V W+B P$ and CAG $(n=1)$, BP and CAG $(n=1)$ and P-PMMA $(n=1)$. In dogs that underwent surgery in two intervertebral spaces, evaluation of each intervertebral space was individual and the level of 
Table 2. Clinical outcome of surgical treatment of the wobbler syndrome in dogs.

\begin{tabular}{|c|c|c|c|c|c|c|c|c|c|}
\hline 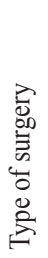 & $\begin{array}{l}\check{b} \\
\stackrel{0}{0}\end{array}$ & 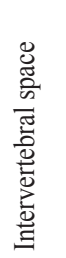 & 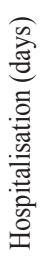 & 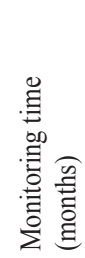 & 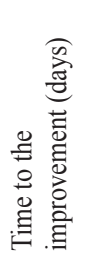 & 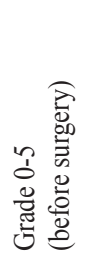 & 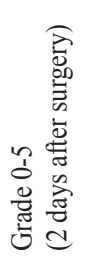 & 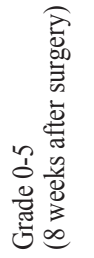 & 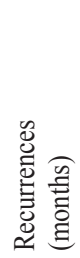 \\
\hline \multirow{10}{*}{ A } & $1 \mathrm{~A}$ & $3-4$ & 6 & 13 & 28 & 4 & 3 & 1 & 11 \\
\hline & 2 & $6-7$ & 6 & 14 & 42 & 4 & 3 & 0 & 14 \\
\hline & 3 & $6-7$ & 3 & $<1$ & DET & 3 & 3 & NDA & \\
\hline & 4 & $3-4$ & 12 & 84 & 7 & 5 & 3 & 1 & \\
\hline & 5 & $6-7$ & \multicolumn{3}{|c|}{ Death in perioperative period } & 5 & NDA & & NDA \\
\hline & 6 & $6-7$ & 3 & 55 & 42 & 3 & 2 & 0 & \\
\hline & 7 & $4-5$ & 2 & 36 & 28 & 4 & 2 & 0 & \\
\hline & 8 & $6-7$ & 5 & 24 & 21 & 4 & 4 & 0 & \\
\hline & 9 & $2-3$ & 3 & 10 & 7 & 4 & 2 & 0 & \\
\hline & 10 & $6-7$ & 5 & 8 & 7 & 1 & 1 & 0 & \\
\hline \multirow{13}{*}{ B } & 11 & $6-7$ & 1 & $<1$ & DET & 2 & 3 & NDA & \\
\hline & $12 \mathrm{~A}$ & $5-6$ & 3 & 42 & 35 & 3 & 2 & 0 & 31 \\
\hline & $12 \mathrm{~B}$ & $6-7$ & 3 & 42 & 35 & 3 & 2 & 0 & \\
\hline & 13 & $6-7$ & 1 & 2 & 14 & 2 & 2 & 0 & \\
\hline & 14 & $6-7$ & 25 & 12 & 28 & 4 & 4 & 0 & 12 \\
\hline & 15 & $6-7$ & 3 & 46 & 7 & 3 & 2 & 0 & \\
\hline & 16 & $5-6$ & 14 & $<1$ & DET & 4 & 5 & NDA & \\
\hline & $17 \mathrm{~A}$ & $5-6$ & 8 & 41 & 28 & 5 & 5 & 0 & \\
\hline & $17 \mathrm{~B}$ & $6-7$ & 8 & 41 & 28 & 5 & 5 & 0 & \\
\hline & 18 & $5-6$ & 12 & 15 & 56 & 5 & 5 & 0 & \\
\hline & $19 \mathrm{~A}$ & $4-5$ & 3 & $<1$ & DET & 5 & 5 & NDA & \\
\hline & $19 \mathrm{~B}$ & $5-6$ & 3 & $<1$ & DET & 5 & 5 & NDA & \\
\hline & 20 & 6-7 & 3 & 12 & 18 & 4 & 4 & 3 & \\
\hline \multirow{4}{*}{$\mathrm{C}$} & $21 \mathrm{~A}$ & $4-5$ & 7 & 6 & 84 & 4 & 5 & 2 & 5 \\
\hline & $21 \mathrm{~B}$ & $5-6$ & 7 & 6 & 84 & 4 & 5 & 2 & \\
\hline & $1 \mathrm{~B}$ & $6-7$ & 6 & 13 & 28 & 4 & 3 & 1 & 11 \\
\hline & 22 & $5-6$ & 2 & NDA & 14 & 2 & 2 & NDA & \\
\hline $\mathrm{D}$ & 23 & $6-7$ & 16 & 2 & DET & 4 & 4 & 5 & \\
\hline $\mathrm{E}$ & 24 & 6-7 & 3 & 7 & 1 & 3 & 1 & 0 & \\
\hline $\mathrm{F}$ & 25 & 6-7 & 5 & 44 & 20 & 4 & 4 & 0 & 44 \\
\hline
\end{tabular}

Type of surgery: A - ventral slot; B - intervertebral washer plus vertebral body screws and PMMA bridge; C - intervertebral washer and cancellous autograft; D - buttress plate and cancellous autograft; E - intervertebral washer plus buttress plate and cancellous autograft; F - smooth pins and PMMA bridge; NDA - no data available; DET - deterioration

clinical severity was common for both intervertebral spaces (Table 2 - dogs identified as subgroups A and B). The success of the surgical treatment of CCSM was defined as a status without any neurological disability evaluated 8 weeks after the surgery with only tolerance of pain expression (grade $0-1$ ). The surgical treatment was evaluated as failed in cases where data were not available because of early euthanasia due to progressive 
deterioration of the neurological status or stagnation (Table 2 - NDA). Success was not evaluated when data were not available for other reasons. For statistical evaluation only those surgical methods were selected that were used to treat CCSM more than $5 \times$, and only complete data were always tested.

In the perioperative period, the incidence of complications associated with surgery was also monitored (excessive bleeding, dysphagia, problematic healing of the surgical wound). Death or euthanasia due to dilated cardiomyopathy or gastric dilatation and volvulus syndrome, postanaestetic complications such as renal failure, a short breakdown of barking, short-term change in the intensity of feeding and watering, and shortterm moderate pain on neck movement were not considered as postoperative complications. The status of dogs was evaluated on the basis of post-operative checks or telephone consultations with the owner. The obtained data were evaluated by descriptive statistical methods. Non-parametric Wilcoxon test for paired data (Kyplot version 2.0beta 15-32bit) was used for statistical evaluation of the results of surgical treatment of CCSM.

\section{Results}

In the period between January 2000 and December 2010, a total of 1,626 dogs with neurological findings were examined and treated at the Department of Surgery and Orthopaedics of the SAC UVPS Brno. Out of the 1,626 dogs, cervical spine lesion was diagnosed in 425 dogs. Out of the 425 dogs with cervical spine lesion, 69 dogs (reference group) suffered from CCSM (16.2\% of dogs with neurological findings in the cervical spine; $4.2 \%$ of dogs with general neurological findings). The mean age and weight of dogs with CCSM in the reference group was $7.1 \pm 2.4$ years and $38.7 \pm 12.0 \mathrm{~kg}$, respectively.

The neurological severity of illness in dogs with CCSM $(n=69)$ on the basis of clinical examination (Table 1) was as follows: grade $0(n=0)$, grade 1 in $13 \%(n=9)$, grade 2 in $27.6 \%(n=19)$, grade 3 in $23.2 \%(n=16)$, grade 4 in $24.6 \%(n=17)$ and grade 5 in $11.6 \%$ $(n=8)$. From the reference group of clinically examined dogs $(n=69)$, complete diagnosis of the CCSM disease as well as evaluation of compression of the spinal cord based on cranial myelography was determined in 48 dogs (69.6\% of the reference group). Simple compression in one intervertebral space was detected in 27 dogs, dual compression in two intervertebral spaces in 14 dogs and multiple compressions in three or more intervertebral spaces in 7 dogs. Overall, compression was detected in 48 dogs in 81 intervertebral spaces $(\mathrm{n}=81)$ with the following localization: $\mathrm{C} 2-\mathrm{C} 3$ in $2.5 \%(\mathrm{n}=2), \mathrm{C} 3-\mathrm{C} 4$ in $13.6 \%(\mathrm{n}=11)$, C4-C5 in $13.6 \%(n=11)$, C5-C6 in $22.2 \%(n=18)$ and C6-C7 in $48.1 \%(n=39)$. The vast majority of the spinal cord compressions (48 compressions) were located on the ventral side $(59.3 \%), 13$ compressions on the dorsal side (16\%), 0 compression on the lateral side $(0.0 \%)$ and combined multilateral compression was detected in 20 cases $(24.7 \%)$. Most of these compressions were dynamic traction responsive (56 compressions, i.e. $69.1 \%$ ) and less frequently compressions were static non-reactive on flexion, extension or application of traction forces (25 compressions, i.e. 30.9\%). In 8 dogs with CCSM detected in more intervertebral spaces simultaneously, combination of static and dynamic types of lesions was found.

The resulting assessment of individual surgical methods is shown in Table 2. In the case of SLOT, a significant difference was found in the severity of clinical symptoms before surgery and 2 days after surgery $(P=0.031)$, and a highly significant difference in the severity of clinical symptoms was found before surgery and 8 weeks after surgery $(P=0.016)$. In case of one dog, progressive bleeding and death occurred during surgery and the dog was excluded from the evaluation of success. Eight of the 9 surgical interventions $(88.9 \%)$ were successfully resolved with this method. By contrast, in the case of IVW + BS-PMMA, no significant difference was found in the severity of clinical symptoms before surgery and 2 days after surgery $(P=0.669)$, but the severity of clinical symptoms was highly significantly different before surgery and 8 weeks after surgery $(P=0.008)$. The method of IVW + BS-PMMA was successfully used in 8 of the 13 surgical interventions $(61.5 \%)$. Using the method of IVW + BS-PMMA, we recorded postoperative complications 
in 3 dogs $(12 \%)$ : excessive bleeding and death in the perioperative period ( 1 dog with SLOT), seroma formation and wound dehiscence (1 dog with BP and CAG), dysphagia and progressive postoperative cachexia (1 dog with IVW+BP and CAG).

In summary, after the surgery of 25 dogs we noted the following outcome: 6 dogs were alive, 5 dogs were euthanized for recurrence of CCSM, 3 dogs were euthanized for unimproved condition, 4 dogs died (gastroduodenal ulcer, dilated cardiomyopathy, anuria, massive bleeding during surgery) and 6 dogs were euthanized for other reasons (geriatric patient, mammary neoplasia, neoplasia of colon, gastric neoplasia, gastric dilatation and volvulus syndrome, dysphagia), and in one dog the data are not documented. Recurrences of CCSM after the surgical treatment were found in 6 dogs in the group of direct and indirect decompression techniques (24\%).

\section{Discussion}

Although the wobbler syndrome in this breed is not so rare, the number of surgically treated patients is actually very small and there is scarcity of relevant data for comparing different methods. Given the average age of dogs with clinically examined CCSM disease (7.1 years in this study), the financial cost and possible accompanying disease, only some dogs undergo complete diagnosis with diagnostic imaging methods $(69.6 \%$ of dogs in our case). Distribution and localization of the spinal cord compression type was similar to literary sources (Da Costa et al. 2012). Almost in half of the cases (43.8\%) disease affected multiple sites of the cervical spine simultaneously. Due to the challenging course of surgical treatment and uncertain prognosis, owners sometimes ask for a conservative treatment only. Conservative treatments with their own protocols of medication are successful up to $54 \%$ of cases with a big disadvantage of the high risk of recurrence of clinical problems (Da Costa et al. 2008; De Decker et al. 2009). In our group of 69 patients with CCSM, 59 dogs $(84.1 \%)$ were primarily treated conservatively. Conservative treatment is usually temporary and when CCSM recurs, the owner requires a surgical method of treatment or euthanasia of the dog. In our record of a total of 25 surgically treated dogs, 14 dogs underwent conservative treatment first without success $(56 \%)$. This delay can have an adverse effect on the overall result of the surgery. In general, success of surgical methods is around 80-90\% (Rusbridge et al. 1998; Da Costa et al. 2008). New trends in the CCSM treatment include minimally invasive surgical methods (Leperlier et al. 2011) and procedures preserving intervertebral motion (A d a m o 2011). We achieved the best results at our Department with two methods that we have found the best for use in routine practice. For the static type of compression we used the SLOT method (88.9\% improvement). Only in one dog there was a stagnation in the postoperative status (postoperative treatment was then added to the NSAID medication within 4 weeks and rise to the gastroduodenal ulcer with fatal consequences). For dynamic compression we observed improvement in 8 weeks in the case of lesions treated by IVW + BS-PMMA (61.5\%). In the case of the IVW + BS-PMMA method we recorded anaesthetic complication in one dog with anuria and progressive deterioration of the neurological status with early mortality 7 days after surgery. Another dog was a Doberman pinscher with severe clinical findings, treated by the IVW + BS-PMMA method in two intervertebral spaces at once, when the owner the dog euthanize three days after the surgery because of too demanding postoperative care and stagnation of neurological status. Based on previous experiences improvement in the condition often occurs after several weeks after surgery (Table 2). It is possible that improvement of the neurological status would have come later and this could negatively overshadow results. In another dog treated by IVW + BS-PMMA a sudden relapse of CCSM occurred approximately 12 months after surgery in connection with surgical treatment of the syndrome of gastric dilatation and volvulus in a private veterinary practice. In this 
case, we could not eliminate secondary damage of the cervical spine stabilization upon manipulation with the dog.

Recurrence of CCSM after surgical treatment was found in six dogs $(24 \%)$. Similar results were published previously (Jeffery and McKee 2001). This finding supports the view that the "domino effect" does not appear only due to fusion of vertebrae and mechanical changes but also as a simple relapse in the most common location, i.e. on the site adjacent to the site of previous surgery irrespective of its type (McKee and Sharp 2002).

Based on the results of our study we conclude that direct ventral slot decompression is useful in clinical cases of the CCSM with static compression, and intervertebral washer plus vertebral body screws and PMMA bridge can be successfully chosen in dogs with dynamic CCSM compression. An in vitro / ex vivo study evaluating the biomechanical aspects of various types of surgical procedures in CCSM may contribute to further extend the knowledge of surgical treatment of CCSM.

\section{Acknowledgments}

This work was supported by the Ministry of Education, Youth and Sport grant intended for institutional research of VFU Brno.

\section{References}

Adamo PF 2011: Cervical arthroplasty in two dogs with disk-associated cervical spondylomyelopathy. J Am Vet Med Assoc 239: 808-817

Da Costa RC 2010: Cervical spondylomyelopathy (wobbler syndrome) in dogs. Vet Clin N Am - Small Anim Pract 40: 881-913

Da Costa RC, Echandi RL, Beauchamp D 2012: Computed tomography myelographic findings in dogs with cervical spondylomyelopathy. Vet Radiol Ultrasound 53: 64-70

Da Costa RC, Parent JM, Holmberg DL, Sinclair D, Monteith G 2008: Outcome of medical and surgical treatment in dogs with cervical spondylomyelopathy: 104 cases (1988-2004). J Anim Vet Med Assoc 233: 1284-1290

De Decker S, Bhatti SFM, Duchateau L, Martle VA, Van Soens I, Van Meervenne SAE, Saunders JH, Van Ham LML 2009: Clinical evaluation of $51 \mathrm{dogs}$ treated conservatively for disc-associated wobbler syndrome. J Small Anim Pract 50: 136-142

Garosi L 2004: The neurological examination. In: Platt SR, Olby NJ: BSAVA Manual of canine and feline neurology, BSAVA, Gloucester, pp. 1-23

Jeffery ND, McKee WM 2001: Surgery for disc-associated wobbler syndrome in the dog - An examination of the controversy. J Small Anim Pract 42: 574-581

Leperlier D, Manassero M, Blot S, Thibaud JL, Viateau V 2011: Minimally invasive video-assisted cervical ventral slot in dogs - A cadaveric study and report of 10 clinical cases. Vet Comp Orthop Traumatol 24: 50-56

McKee WM, Butterworth SJ, Scott HW 1999: Management of cervical spondylopathy-associated intervertebral disc protrusions using metal washers in 78 dogs. J Small Anim Pract 40: 465-472

McKee WM, Sharp JH 2002: Cervical spondylopathy. In: Slatter D: Textbook of Small Animal Surgery. W. B. Saunders, Philadelphia, 3rd ed., pp. 1180-1193

Robertson I, Thrall DE 2011: Imaging dogs with suspected disc herniation: Pros and cons of myelography, computed tomography, and magnetic resonance. Vet Radiol Ultrasound 52 Supp. 1: S81-S84

Rusbridge C, Wheeler SJ, Torrington AM, Pead MJ, Carmichael S 1998: Comparison of two surgical techniques for the management of cervical spondylomyelopathy in dobermanns. J Small Anim Pract 39: 425-431

Sharp NJH, Wheeler SJ 2005: Cervical spondylomyelopathy. In: Sharp NJH, Wheeler SJ: Small animal spinal disorders. Diagnosis and surgery. Elsevier, 2nd ed., pp. 211-246

Sharp NJH, Wheeler SJ, Cofone M 1992: Radiological evaluation of "wobbler" syndrome - caudal cervical spondylomyelopathy. J Small Anim Pract 33: 491-499 